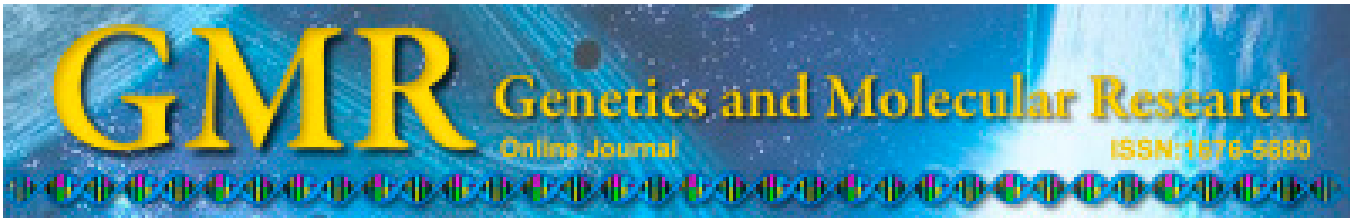

\title{
IDH1 and $I D H 2$ mutations in different histologic subtypes and WHO grading gliomas in a sample from Northern Brazil
}

\author{
I.A. Pessôa ${ }^{1,2}$, F.E.S. Sagica ${ }^{2}$, N.P. Anselmo ${ }^{3}$, J.R.N. Brito ${ }^{4}$ and \\ E.H.C. de Oliveira ${ }^{2,5}$ \\ ${ }^{1}$ Programa de Pós-Graduação em Genética e Biologia Molecular, \\ Universidade Federal do Pará, Belém, PA, Brasil \\ ${ }^{2}$ Laboratório de Cultura de Tecidos e Citogenética, Instituto Evandro Chagas, \\ Ananindeua, PA, Brasil \\ ${ }^{3}$ Laboratório de Biologia Molecular Francisco Mauro Salzano, \\ Universidade Federal do Pará, Belém, PA, Brasil \\ ${ }^{4}$ Programa de Pós-Graduação em Neurociências e Biologia Celular, \\ Universidade Federal do Pará, Belém, PA, Brasil \\ ${ }^{5}$ Faculdade de Ciências Naturais, ICEN, Universidade Federal do Pará, \\ Belém, PA, Brasil \\ Corresponding author: E.H.C. de Oliveira \\ E-mail: ehco@ufpa.br / edivaldodeoliveira@iec.pa.gov.br
}

Genet. Mol. Res. 14 (2): 6533-6542 (2015)

Received August 18, 2014

Accepted January 19, 2015

Published June 12, 2015

DOI http://dx.doi.org/10.4238/2015.June.12.6

\begin{abstract}
Glioma is a term used to describe tumors derived from glial cells. These tumors are divided into subgroups based on the histological morphology and similarity of their differentiated glia cells. Traditionally, they are classified according to the World Health Organization and include astrocytomas, oligodendrogliomas, ependymomas, and oligoastrocytomas. Like most cancers, gliomas develop as a result of genetic changes that accumulate with tumor progression. Alterations in isocitrate dehydrogenase 1 (IDH1) and IDH2 were found to be relevant in the classification and prognostic of gliomas. Because of the importance of mutations in these genes, particularly in
\end{abstract}


$I D H 1$, in different proposals of the genesis and progression of gliomas, we analyzed the occurrence of mutations in these genes in samples obtained from patients from Belém (PA, Brazil) using polymerase chain reaction-single-strand conformation polymorphism followed by sequencing. We compared the results obtained from tumors of different malignancy grades, evaluating the significance of the associations between different variables. R132H was the only mutation found in $17.6 \%(6 / 34)$ of cases, including in astrocytomas, anaplastic astrocytomas, oligodendroglioma, and anaplastic oligoastrocytoma. No mutations were found in the $I D H 2$ gene. We found no significant relationship between the identified mutations in $I D H 1$ and the variables. Our data could not confirm that mutations in IDH1/IDH2 are indicative of malignancy and prognosis. However, the results support that the mutation in $I D H 1$ gene was an early event in the development of gliomas, as it was found in tumors of different malignancy grades.

Key words: Isocitrate dehydrogenase 1; Isocitrate dehydrogenase 2; R132H; Glioma; Direct DNA sequencing;

Single-strand conformation polymorphism

\section{INTRODUCTION}

Gliomas are the most frequent primary brain tumors and include a variety of histological types. Most glioma classifications rely on the morphological similarities of the tumor cells with non-neoplastic glial cells based on the recommendations of the World Health Organization (WHO) (Louis et al., 2007): astrocytic, oligodendroglial, mixed oligoastrocytic, or ependymal tumors. New molecular markers have emerged in recent decades and have been shown to be useful as predictive, prognostic, and, in some cases, diagnostic markers (Gupta and Salunke, 2012).

For example, mutations in the isocitrate dehydrogenase 1 (IDH1) gene were originally identified by Parsons et al. (2008) in 22 glioblastomas that had been subjected to nextgeneration sequencing technologies. The glioblastomas showing $I D H 1$ mutations occurred in a large fraction of young patients and in most patients with secondary glioblastomas and were associated with higher survival rates. IDH1, IDH2, and IDH3 are enzymes involved in the citric acid cycle and catalyze the oxidative decarboxylation of isocitrate to $\alpha$-ketoglutarate while reducing $\mathrm{NADP}^{+}$to NADPH $\left(\mathrm{NAD}^{+}\right.$to NADH in the case of IDH3) (Kim and Liau, 2012). IDH1 is found within the cytoplasm and peroxisomes, whereas IDH2 and IDH3 are localized solely in the mitochondria (Kim and Liau 2012).

Later, multiple studies corroborated these findings and additionally revealed that somatic IDH1 mutations are present in the vast majority of low-grade diffuse (WHO grade II) and anaplastic (WHO grade III) astrocytic, oligodendroglial, and mixed oligodendroglial neoplasms (Balss et al., 2008; Nobusawa et al., 2009; Yan et al., 2009). Mutations in IDH2 have also been identified in gliomas, although they are much less common and are mutually exclusive with mutations in IDH1 (Hartmann et al., 2009; Yan et al., 2009; Sonoda et al., 2009). IDH3 mutations have not been implicated in neoplasias (Reitman and Yan, 2010).

The most common IDH1 mutation is a heterozygous missense mutation that changes 
a guanine to adenine at position 395 in the coding region (c.395G $>$ A), leading to the replacement of arginine by histidine at codon $132(\mathrm{R} 132 \mathrm{H})$ at the enzymatic active site (Zhao et al., 2009). Similarly, IDH2 mutations in gliomas include substitutions at amino acid 172, which is functionally equivalent to amino acid 132 of IDHI (Mukasa et al., 2012).

The mechanisms by which mutations in $I D H$ genes mediate gliomagenesis are currently unknown. However, it has been shown that mutant IDH1 and IDH2 proteins exhibit a gain-offunction phenotype by generating (R)-2-hydroxyglutarate, a toxic metabolite associated with an increased risk of malignant brain tumors in patients with inherited errors of (R)-2-hydroxyglutarate metabolism (Dang et al., 2009). Similarly, $I D H 1$ and $I D H 2$ mutations dramatically increase (R)-2-hydroxyglutarate in acute myeloid leukemia (Gross et al., 2010; Ward et al., 2010). Hence, it is thought that mutations in $I D H 1$ and $I D H 2$ can provide new insights into anti-cancer therapies because of their specificity and metabolic effects (Fu et al., 2010).

Because of the importance of these mutations in gliomas and the absence of such data in the population from the North of Brazil, the aim of this study was to analyze the occurrence of mutations in codons 132 and 172 in the $I D H 1$ and $I D H 2$ genes (corresponding to exon 4 in both genes) in 34 samples of gliomas collected from patients from Belém (PA, Brazil). We also describe their frequencies and possible associations with variables such age, gender, histological types, and WHO histological grading.

\section{MATERIAL AND METHODS}

\section{Patients and tissue samples}

This study was submitted and approved by the Ethics Committee of CCS/UFPA. Tumor samples were obtained fresh at the time of surgery at the Hospital Ophir Loyola (Belém, PA, Brazil). All cases were histologically classified and graded according to the current WHO classification for tumors of the central nervous system. All patients signed a consent form approving the use of the biopsies for scientific purposes. We examined a total of 34 gliomas, including 4 pilocytic astrocytomas (WHO grade I), 1 pilomyxoid astrocytoma (WHO grade II), 3 diffuse astrocytomas (WHO grade II), 3 anaplastic astrocytomas (WHO grade III), 9 gliobastomas (WHO grade IV), 2 gliosarcomas (WHO grade IV), 2 oligodendrogliomas (WHO grade II), 1 oligoastrocytoma (WHO grade II), 3 anaplastic oligoastrocytomas (WHO grade III), 3 ependymomas (WHO grade II), and 3 anaplastic ependymomas (WHO grade III). Clinical parameters including age and gender were collected.

\section{Single-strand conformational polymorphism (SSCP) analysis and direct DNA sequencing for IDH1/IDH2 mutations}

Genomic DNA was extracted from tumor tissue using standard procedures (phenol/ chloroform and ethanol precipitation) and dissolved in elution buffer (10 mM Tris-Cl, $\mathrm{pH} 7.5$, and $1 \mathrm{mM}$ ethylenediaminetetraacetic acid) and stored at $4^{\circ} \mathrm{C}$. Two sets of primers were generated to screen for the presence of point mutations using the SSCP technique. The genomic regions spanning the catalytic domain of IDH1, including codon 132 (exon 4), and of IDH2, including codon 172 (exon 4), were analyzed. Initially, polymerase chain reaction (PCR) amplification was performed on exon 4 of the IDHI gene and on exon 4 of the $I D H 2$ gene. Table 1 shows the primers used for these genes. 
PCR conditions for the $I D H$ genes were: initial denaturation at $95^{\circ} \mathrm{C}$ for $5 \mathrm{~min}, 35$ cycles at $95^{\circ} \mathrm{C}$ for $1 \mathrm{~min}, 59^{\circ}-60^{\circ} \mathrm{C}$ for $1 \mathrm{~min}$, and $72^{\circ} \mathrm{C}$ for $50 \mathrm{~s}$, with a final extension of 8 min at $72^{\circ} \mathrm{C}$. After amplification, the PCR products were loaded onto $10 \%$ non-denaturing polyacrylamide gels (without glycerol), electrophoresed at $220 \mathrm{~V}$ for $8 \mathrm{~h}$, and then silverstained. Samples displaying an altered PCR-SSCP pattern were re-amplified by PCR with the same set of primers, and the PCR products were sequenced using the Big Dye Terminator kit (v3.1 - Applied Biosystems, Foster City, CA, USA) following manufacturer specifications. The ABI PRISM 3130 Genetic Analyzer (Applied Biosystems) was utilized to read the sequences. The software programs Chromas Lite (v2.1.1) and BioEdit (v7.2.5) were used for sequence analysis.

Table 1. Primers used for the $I D H 1$ and $I D H 2$ genes.

\begin{tabular}{llcl}
\hline Gene & Primer & Amplified fragment & References \\
\hline IDH1 & F - 5'-CGGTCTTCAGAGAAGCCATT-3' & 172 bp & Metellus et al. (2010) \\
& R - 5'-CACATACAAGTTGGAAATTCTGG-3' & & \\
IDH2 & F - 5'-AGCCCATCATCTGCAAAAAC-3' & 182 bp & Badiali et al. (2012). \\
& R - 5'-TGTGGCCTTGTACTGCAGAG-3' & & Hartmann et al. (2009) \\
\hline
\end{tabular}

\section{Statistical analysis}

Microsoft Excel and Graph Pad Prism 5.0 were used to store information in the form of databases and to apply descriptive statistics, respectively. Parametric statistics tests (analysis of variance) and non-parametric statistics tests (Fisher exact test, Q-Cochran analysis of variance) were used to compare the frequencies and mean scores of each group, respectively, noting possible statistical differences. Additionally, Pearson correlation and the contingency coefficient were evaluated to reveal associations between variables in the samples.

\section{RESULTS}

\section{Study population}

Among the 34 gliomas analyzed, 19 (55.9\%) were from males and 15 (44.1\%) were from females. The median and mean ages of the patients were 42.5 and 37.1 years, respectively, ranging 1-74 years in the total sample. When comparing the age distribution among the different grades of malignancy by analysis of variance, we found statistically significant differences in younger patients with less aggressive tumors, whereas the mean age was accompanied by an increase in tumor aggressiveness (Table 2).

Table 2. Comparison of WHO grading $\mathrm{x}$ age by ANOVA and the Tukey test.

\begin{tabular}{lcl}
\hline WHO histological grading & Median age & Significance (P value) \\
\hline I & 11.8 & Median I and II (P $>0.05)$ \\
& & Median I and III (P $<0.01)$ \\
II & 16.4 & Median I and IV (P $<0.01)$ \\
& & Median II and III (P $<0.01)$ \\
III & 51.3 & Median II and IV (P $<0.01)$ \\
IV & 56.4 & Median III and IV (P $>0.05)$ \\
\hline
\end{tabular}




\section{IDH1/IDH2 status}

PCR-SSCP analysis revealed aberrantly migrated bands from the IDH1 gene in 6 (17.6\%) samples (Table 3), while no abnormal migration patterns were observed in the IDH2 gene. The results for each gene are shown in Figure 1. The 6 samples with abnormal IDHI migration patterns were sequenced to determine the types of mutations present.

Table 3. $I D H 1$ mutated gliomas.

\begin{tabular}{|c|c|c|c|c|}
\hline Sample & Histopathological & WHO grading & Gender & Age \\
\hline CNS 21 & Anaplastic oligoastrocytoma & III & $\mathrm{M}$ & 42 \\
\hline CNS 31 & Anaplastic astrocytoma & III & $\mathrm{F}$ & 65 \\
\hline CNS 44 & Anaplastic astrocytoma & III & M & 60 \\
\hline CNS 88 & Anaplastic oligoastrocytoma & III & $\mathrm{F}$ & 63 \\
\hline CNS 96 & Oligodendroglioma & II & $\mathrm{F}$ & 43 \\
\hline CNS 126 & Diffuse astrocytoma & II & $\mathrm{F}$ & 34 \\
\hline
\end{tabular}
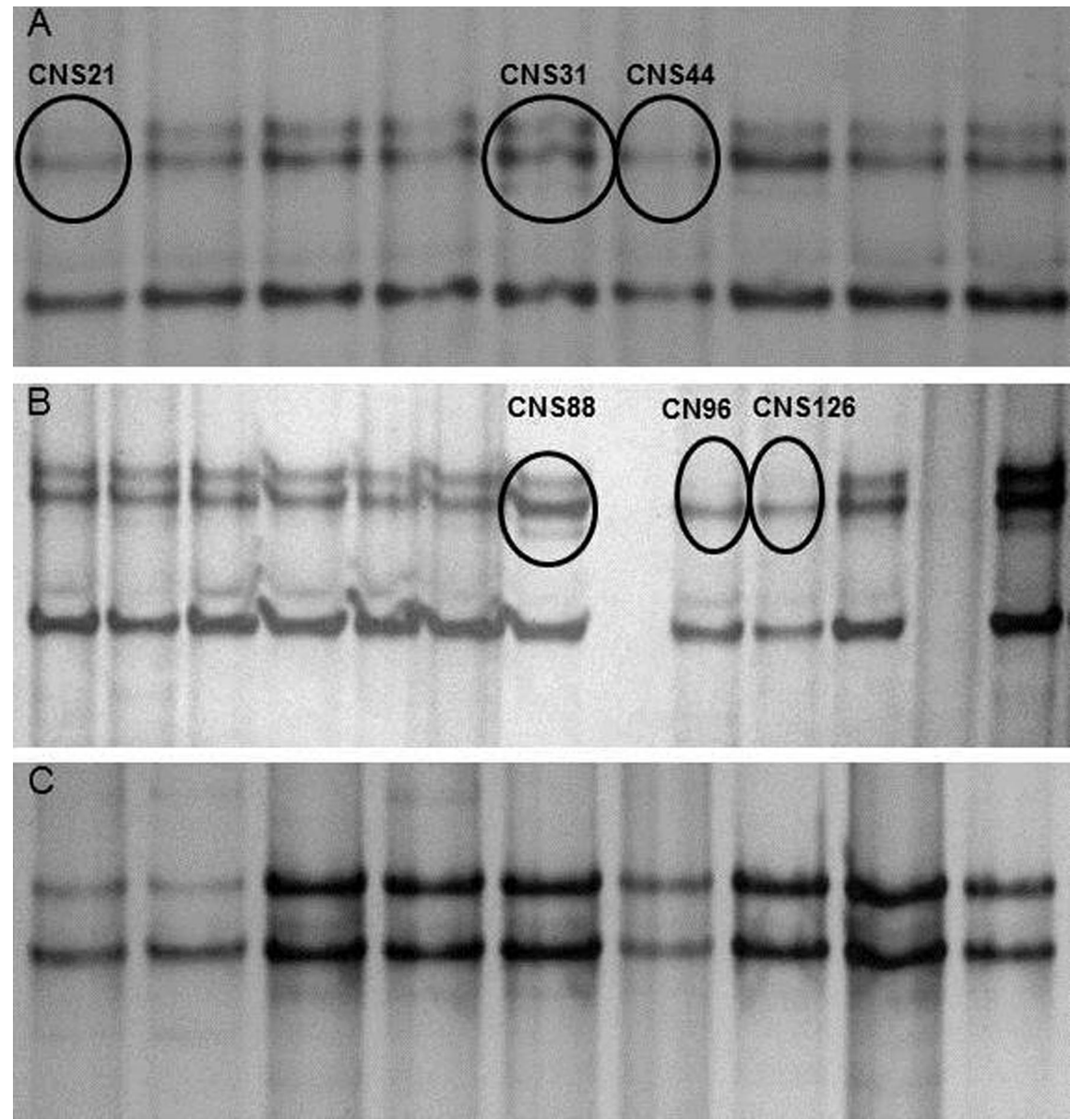

Figure 1. A. B. Exon 4 of $I D H 1$ gene SSCP. Circles indicate samples with different mobility. C. Exon 4 of $I D H 2$ gene SSCP. All samples showed the same pattern of mobility. 
Sequence evaluation of exon 4 in the $I D H 1$ gene revealed heterozygous mutations in the 6 cases analyzed in codon 132. All detected mutations were missense mutations. The transition $\mathrm{G}>\mathrm{A}$ at position 395 of the coding region resulted in substitution of the amino acid arginine with histidine (R132H) and was identified in all cases (Figure 2). The frequencies of genotypes GG (Arg/Arg), GA (Arg/His), and AA (His/His) were 0.824 (28/34), $0.176(6 / 34)$, and $0.000(0 / 34)$, respectively. The observed allele frequency for $\mathrm{G}$ was 0.912 , while for $\mathrm{C}$ this value was 0.088 . Therefore, the distribution of these genotypes and allele frequencies agreed with those calculated based on Hardy-Weinberg equilibrium $(\mathrm{P}=0.9231)$.

The $\mathrm{R} 132 \mathrm{H}$ mutation was more prevalent in female patients $(66.7 \%)$ and the mean age of patients with this mutation was 51.2 years. However, no significant association was found between the presence of this mutation and gender and age $(\mathrm{P}=0.1101$ and $\mathrm{P}=0.1316$, respectively). The mutation mainly affected astrocytomas (50\%), although no statistically significant difference was identified among all histologic subtypes studied $(\mathrm{P}=0.1116)$. In addition, no association was observed between the presence of the R132H mutation and WHO grading ( $\mathrm{P}$ $=0.0748$ ), although $66.7 \%$ of the mutations occurred in WHO grade III gliomas.

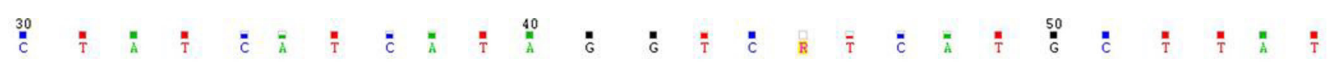

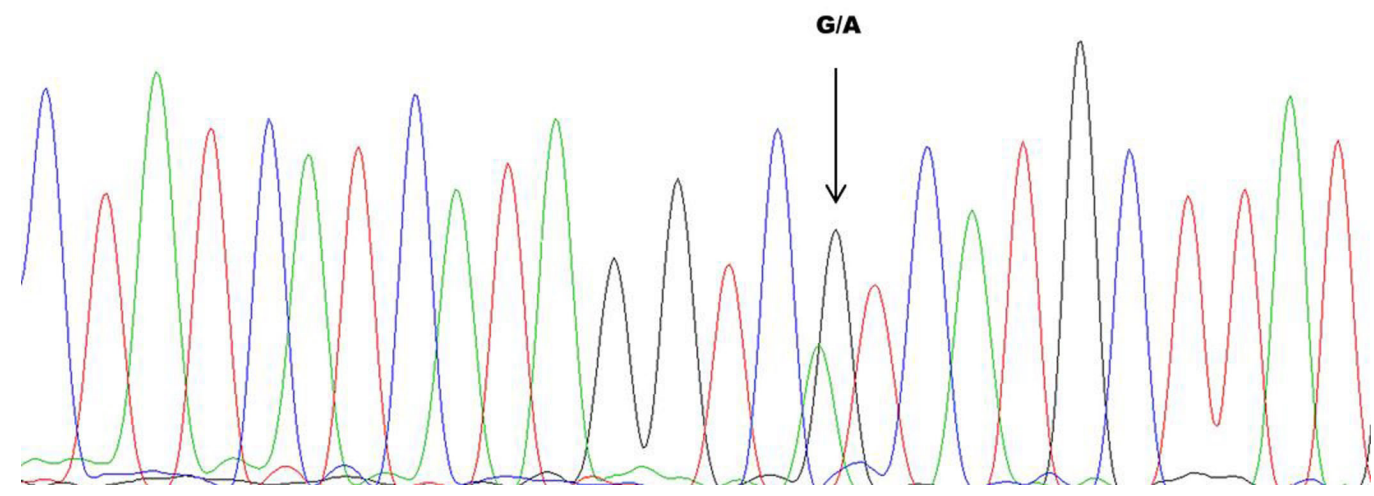

Figure 2. Nucleotide changes identified in $I D H 1$. The arrow shows the transition G>A in codon 132 (Arg/His).

\section{DISCUSSION}

A more detailed molecular understanding of specific biomarkers can reduce the problems of subjectivity in histological assessment and incomplete tissue sampling (Hartmann et al., 2010). Therefore, it is important to improve the classification of gliomas to better stratify patients in clinical trials, better predict outcome, and provide specific, tailored therapy (Gupta and Salunke, 2012). In recent years, it was observed that genetic mutations frequently occur in key enzymes that regulate important metabolic pathways (Dang et al., 2009; Vander Heiden et al., 2009). Changes in fumarate hydratase and succinate dehydrogenase in hereditary leiomyomatosis, renal cell carcinoma, and paraganglioma were the earliest known examples of mutations in genes encoding mitochondrial enzymes involved 
in metabolism (Frezza et al., 2011; Gill, 2012). The first discovered mutations in the genes encoding isocitrate dehydrogenases (including $I D H 1$ and $I D H 2$ ) have been identified in colon cancer (Sjoblom et al., 2006). In 2008, it was observed that IDHI and IDH2 were mutated in low-grade gliomas and a subset of secondary glioblastomas (Parsons et al., 2008). This discovery led to the rapid development of new concepts in gliomagenesis, and has implicated the Krebs cycle and metabolic pathways in neoplasias.

All IDH1/2 mutations described in the literature are missense mutations (Yan et al., 2009; Ichimura et al., 2009; Hartmann et al., 2009; Agarwal et al., 2013; Yao et al., 2013). Nearly all IDHI mutations in gliomas occur at codon 132, which encodes arginine (R132) within the enzyme's substrate-binding site, with a very rare exception in which arginine 100 is replaced with glutamine (R100Q) in a few tumors (Pusch et al., 2011; Agarwal et al., 2013; Yao et al., 2013). Approximately $90 \%$ of all $I D H 1$ mutations are G to A transitions at the second nucleotide of codon 132 (c.395G $>$ A), resulting in the substitution of arginine with histidine (R132H). Other mutations of IDH1 are far less common but also affect R132, resulting in the substitution of arginine with cysteine (R132C; frequency 3.6-4.6\%), serine (R132S; 0.8$2.5 \%$ ), glycine (R132G; 0.6-3.8\%), or leucine (R132L; 0.5-4.3\%) (Yan et al., 2009; Ichimura et al., 2009; Hartmann et al., 2010; Weller et al., 2011).

Our results confirm these data, as $\mathrm{R} 132 \mathrm{H}$ was the only amino acid sequence alteration in $I D H 1$, accounting for $100 \%$ of the detected mutations. The discrepancies in the literature regarding the low frequencies of R132C, R132S, R132G, and R132L may result from differences in the tumor subtype analyzed and sample size included in the present study. Our analyses also showed that the mean age of patients without IDH1 mutations was lower (34.0357) than that of patients with IDH1 mutations (51.1667), although no significant difference was observed $(\mathrm{P}=0.1316)$. In contrast to these results, Parsons et al. (2008) noted that the mean age of patients with gliobastomas presenting IDH1 mutations was 33 years, whereas the group of patients without $I D H 1$ mutations was 53 years of age. Watanabe et al. (2009) found that patients with low-grade diffuse astrocytomas carrying $I D H 1$ mutations were significantly younger (36.2 \pm 9.4 years) than those without IDH1 mutations $(51.6 \pm 19.6$ years; $\mathrm{P}=0.0004)$, while the mean ages of oligodendroglioma patients with and without $I D H 1$ mutations were not significantly different $(42.6 \pm 12.3$ vs $37.1 \pm 13.1$ years; $\mathrm{P}=0.307$ ). The differences observed between these findings and our data likely occurred because 3 of the 6 patients in this study with mutations were older than 60 years, increasing the mean age of this group. Moreover, among patients without mutations, the sample comprised a substantial number of tumors that typically occur in young individuals, such as pilocytic astrocytomas, diffuse astrocytomas, and WHO grade II ependymomas, reducing the mean age in this group.

Because tumors without $I D H 1$ mutations may contain genetic alterations in codon 172 of the $I D H 2$ gene, the samples were also analyzed by SSCP for $I D H 2$. However, no mutation was identified. These results corroborate data obtained by several previous studies (Yan et al., 2009; Hartmann et al., 2009, 2010; Yao et al., 2013). Summarized data of a series of studies revealed that only 1 of 360 primary glioblastomas harbored a mutation in $I D H 2$, while fewer than $2 \%$ of WHO grades II and III astrocytomas exhibited mutations in this gene, and no more than $6 \%$ of oligodendrogliomas and oligoastrocytomas showed this mutation (Dunn et al., 2013). 
Many studies detected $I D H 1$ mutations at high frequency in secondary glioblastomas $(50-88 \%)$. In contrast, these mutations rarely occur in primary or de novo glioblastomas (3-21\%) (Kim and Liau, 2012; Zhang et al., 2013; Dunn et al., 2013). IDH1 mutations were further identified in the majority of WHO grade II diffuse astrocytoma (59-100\%) and WHO grade III anaplastic astrocytomas (approximately 60\%), oligodendrogliomas (67-93\%), anaplastic oligodendrogliomas (49-86\%), and mixed oligoastrocytomas (50-100\%). The mutation rate in pilocytic astrocytomas, ependymal tumors, or other less common glial tumors are lower (Kim and Liau, 2012; Zhang et al., 2013; Dunn et al., 2013). The frequency of R132H mutations in gliomas in our series was close to the spectrum described above, as we found this mutation in diffuse astrocytomas $(33.3 \% ; 1 / 3)$, anaplastic astrocytoma $(66.6 \% ; 2 / 3)$, oligodendroglioma $(50 \% ; 1 / 2)$, and anaplastic oligoastrocytoma $(66.6 \% ; 2 / 3)$. However, no alterations were identified in samples classified as glioblastoma. This difference may be attributed to the small numbers of particular subtypes of gliomas in other studies. In addition, no mutations were identified in the pilocytic astrocytomas and ependymomas analyzed, confirming data from several studies.

The presence of $I D H$ mutations may be useful for distinguishing astrocytomas and oligodendrogliomas from other central nervous system tumors (Horbinski et al., 2009; Capper et al., 2011). In the future, the $I D H$ mutation status may be incorporated into the existing diagnostic criteria along with the total $1 \mathrm{p} / 19 \mathrm{q}$ loss in oligodendrogliomas or $B R A F$ fusion gene in pilocytic astrocytomas (Riemenschneider et al., 2010). Furthermore, immunohistochemistry analysis of IDH1 R132H is valuable for histological assessment of small and or/crushed biopsy specimens, as well as in the differential diagnosis of glioma types (Gupta et al., 2011). The histopathological utility of IDH1-R132H antibodies, which recognize the mutant protein, has extended to additional clinical scenarios such as the discrimination between diffuse astrocytoma and reactive astrocytosis when combined with a panel of key molecular features (Camelo-Piragua et al., 2011).

WHO grade III tumors showed the highest frequency of the R132H mutation in the present study, and $66.7 \%$ (4/6) of patients had this mutation. However, no significant association between the presence of the $\mathrm{R} 132 \mathrm{H}$ mutation and WHO grading was observed $(\mathrm{P}=0.0748) . I D H 1$ mutations may be an early event in the progression of gliomas, corroborating the findings of other studies (Watanabe et al., 2009; Ichimura et al., 2009). Watanabe et al. (2009) analyzed multiple biopsies from the same patient and reported that most of the samples contained both IDH1 and TP53 mutations (63\% of WHO grade II diffuse astrocytomas) or loss of heterozygosity in 1p/19q (64\% of oligodendrogliomas). In addition, there were no cases in which an $I D H 1$ mutation occurred after the acquisition of either a TP53 mutation or loss of 1p/19q, suggesting that $I D H 1$ mutations are very early events in gliomagenesis and may affect a common glial precursor cell population (Watanabe et al., 2009).

In summary, our results confirmed the presence of IDH1 mutations in diffuse astrocytomas, anaplastic astrocytomas, oligodendroglioma, and anaplastic oligoastrocytoma and showed that only the R132H mutation was present, hence occurring in low-grade gliomas (WHO grades I and II) and high-grade gliomas (WHO grades III and IV), suggesting that they are early events in the progression of gliomas. In contrast, no IDH2 mutations were identified in these tumors. 


\section{ACKNOWLEDGMENTS}

The authors would like to thank Hospital Ophir Loyola, Instituto Evandro Chagas and CNPq for support.

\section{REFERENCES}

Agarwal S, Sharma MC and Jha P (2013). Comparative study of IDH1 mutations in gliomas by immunohistochemistry and DNA sequencing. Neuro. Oncol. 15: 718-726.

Badiali M, Gleize V, Paris S, Moi L, et al. (2012). KIAA1549-BRAF Fusions and IDH Mutations Can Coexist in Diffuse Gliomas of Adults. Brain Pathol. 22: 841-847.

Balss J, Meyer J, Mueller W, Korshunov A, et al. (2008). Analysis of the IDH1 codon 132 mutation in brain tumors. Acta Neuropathol. 116: 597-602.

Camelo-Piragua S, Jansen M, Ganguly A, Kim JC, et al. (2011). A sensitive and specific diagnostic panel to distinguish diffuse astrocytoma from astrocytosis: chromosome 7 gain with mutant isocitrate dehydrogenase 1 and p53. $J$. Neuropathol. Exp. Neurol. 70: 110-115.

Capper D, Reuss D, Schittenhelm J, Hartmann C, et al. (2011). Mutation-specific IDH1 antibody differentiates oligodendrogliomas and oligoastrocytomas from other brain tumors with oligodendroglioma-like morphology. Acta Neuropathol. 121: 241-252.

Dang L, White DW, Gross S, Bennett BD, et al. (2009). Cancer-associated IDH1 mutations produce 2-hydroxyglutarate. Nature 462: 739-744.

Dunn GP, Andronesi OC and Cahill DP (2013). From genomics to the clinic: biological and translational insights of mutant IDH1/2 in glioma. Neurosurg. Focus 34: E2.

Frezza C, Zheng L, Folger O, Rajagopalan KN, et al. (2011). Haem oxygenase is synthetically lethal with the tumour suppressor fumarate hydratase. Nature 477: 225-228.

Fu Y, Huang R, Du J, Yang R, et al. (2010). Glioma-derived mutations in IDH: from mechanism to potential therapy. Biochem. Biophys. Res. Commun. 397: 127-130.

Gill AJ (2012). Succinate dehydrogenase (SDH) and mitochondrial driven neoplasia. Pathology 44: 285-292.

Gross S, Cairns RA, Minden MD, Driggers EM, et al. (2010). Cancer-associated metabolite 2-hydroxyglutarate accumulates in acute myelogenous leukemia with isocitrate dehydrogenase 1 and 2 mutations. J. Exp. Med. 207: 339-344.

Gupta K and Salunke P (2012). Molecular markers of glioma: an update on recent progress and perspectives. J. Cancer Res. Clin. Oncol. 138: 1971-1981.

Gupta R, Webb-Myers R and Flanagan S (2011). Isocitrate dehydrogenase mutations in diffuse gliomas: clinical and aetiological implications. J. Clin. Pathol. 64: 835-844.

Hartmann C, Meyer J, Balss J, Capper D, et al. (2009). Type and frequency of IDH1 and IDH2 mutations are related to astrocytic and oligodendroglial differentiation and age: a study of 1,010 diffuse gliomas. Acta Neuropathol. 118: 469-474.

Hartmann C, Hentschel B, Wick W, Capper D, et al. (2010). Patients with IDH1 wild type anaplastic astrocytomas exhibit worse prognosis than IDH1-mutated glioblastomas, and IDH1 mutation status accounts for the unfavorable prognostic effect of higher age: implications for classification of gliomas. Acta Neuropathol. 120: 707-718.

Horbinski C, Kofler J and Kelly LM (2009). Diagnostic use of IDH1/2 mutation analysis in routine clinical testing of formalin-fixed, paraffin-embedded glioma tissues. J. Neuropathol. Exp. Neurol. 68: 1319-1325.

Ichimura K, Pearson DM, Kocialkowski S, Bäcklund LM, et al. (2009). IDH1 mutations are present in the majority of common adult gliomas but are rare in primary glioblastomas. Neuro. Oncol. 11: 341-347.

Kim W and Liau LM (2012). IDH mutations in human glioma. Neurosurg. Clin. N. Am. 23: 471-480.

Louis DN, Ohgaki H, Wiestler OD and Cavenee WK (2007). WHO classification of tumors of the central nervous systems, 4th edn. International Agency for Research on Cancer (IARC), Lyon.

Metellus P, Coulibaly B, Colin C, De Paula AM, et al. (2010). Absence of IDH mutation identifies a novel radiologic and molecular subtype of WHO grade II gliomas with dismal prognosis. Acta Neuropathol. 120: 719-729.

Mukasa A, Takayanagi S and Saito K (2012). Significance of IDH mutations varies with tumor histology, grade, and genetics in Japanese glioma patients. Cancer Sci. 103: 587-592.

Nobusawa S, Watanabe T, Kleihues P and Ohgaki H (2009). IDH1 mutations as molecular signature and predictive factor of secondary glioblastomas. Clin. Cancer Res. 15: 6002-6007.

Parsons DW, Jones S, Zhang X, Lin JC, et al. (2008). An integrated genomic analysis of human glioblastoma multiforme. Science 321: 1807. 
Pusch S, Sahm F, Meyer J, Mittelbronn M, et al. (2011). Glioma IDH1 mutation patterns off the beaten track. Neuropathol. Appl. Neurobiol. 37: 428-430.

Reitman ZJ and Yan H (2010). Isocitrate dehydrogenase 1 and 2 mutations in cancer: alterations at a crossroads of cellular metabolism. J. Natl. Cancer Inst. 102: 932-941.

Riemenschneider MJ, Jeuken JW, Wesseling P, Reifenberger G, et al. (2010). Molecular diagnostics of gliomas: state of the art. Acta Neuropathol. 120: 567-584.

Sjoblom T, Jones S, Wood LD, Parsons DW, et al. (2006). The consensus coding sequences of human breast and colorectal cancers. Science 314: 268-274.

Sonoda Y, Kumabe T, Nakamura T, Saito R, et al. (2009). Analysis of IDH1 and IDH2 mutations in Japanese glioma patients. Cancer Sci. 100: 1996-1998.

Vander Heiden MG, Cantley LC and Thompson CB (2009). Understanding the Warburg effect: the metabolic requirements of cell proliferation. Science 324: 1029-1033.

Ward PS, Patel J, Wise DR, Abdel-Wahab O, et al. (2010). The common feature of leukemia-associated IDH1 and IDH2 mutations is a neomorphic enzyme activity converting a-ketoglutarate to 2-hydroxyglutarate. Cancer Cell 17: 225234.

Watanabe T, Nobusawa S and Kleihues P (2009). IDH1 mutations are early events in the development of astrocytomas and oligodendrogliomas. Am. J. Pathol. 174: 1149-1153.

Weller M, Wick W and von Deimling A (2011). Isocitrate dehydrogenase mutations: a challenge to traditional views on the genesis and malignant progression of gliomas. Glia 59: 1200-1204.

Yan H, Parsons DW, Jin G, McLendon R, et al. (2009). IDH1 and IDH2 mutations in gliomas. N. Engl. J. Med. 360: 765-773.

Yao Y, Chan AK, Qin ZY, Chen LC, et al. (2013). Mutation analysis of IDH1 in paired gliomas revealed IDH1 mutation was not associated with malignant progression but predicted longer survival. PLoS One 8: e67421.

Zhang C, Moore LM, Li X, Yung WK, et al. (2013). IDH1/2 mutations target a key hallmark of cancer by deregulating cellular metabolism in glioma. Neuro. Oncol. 15: 1114-1126.

Zhao S, Lin Y, Xu W, Jiang W, et al. (2009). Glioma-derived mutations in IDH1 dominantly inhibit IDH1 catalytic activity and induce HIF-1alpha. Science 324: 261-265. 